\title{
Symplastic Continuity between Companion Cells and the Translocation Stream: Long-Distance Transport Is Controlled by Retention and Retrieval Mechanisms in the Phloem ${ }^{1}$
}

\author{
Brian G. Ayre*, Felix Keller, and Robert Turgeon \\ Department of Plant Biology, Cornell University, Ithaca, New York 14853 (B.G.A., R.T.); and Institute of \\ Plant Biology, University of Zurich, CH-8008 Zurich, Switzerland (F.K.).
}

\begin{abstract}
Substantial symplastic continuity appears to exist between companion cells (CCs) and sieve elements of the phloem, which suggests that small solutes within the CC are subject to indiscriminate long-distance transport via the translocation stream. To test this hypothesis, the distributions of exotic and endogenous solutes synthesized in the CCs of minor veins were studied. Octopine, a charged molecule derived from arginine and pyruvate, was efficiently transported through the phloem but was also transferred in substantial amounts to the apoplast, and presumably other non-phloem compartments. The disaccharide galactinol also accumulated in non-phloem compartments, but long-distance transport was limited. Conversely, sucrose, raffinose, and especially stachyose demonstrated reduced accumulation and efficient transport out of the leaf. We conclude that small metabolites in the cytosol of CCs do enter the translocation stream indiscriminately but are also subject to distributive forces, such as nonselective and carrier-mediated membrane transport and symplastic dispersal, that may effectively clear a compound from the phloem or retain it for long-distance transport. A model is proposed in which the transport of oligosaccharides is an adaptive strategy to improve photoassimilate retention, and consequently translocation efficiency, in the phloem.
\end{abstract}

Companion cells (CCs) and sieve elements (SEs) have a distinctly close relationship, and together form the SE/CC complex (SECCC). Mature SEs are enucleate, devoid of all but a few diminutive organelles, and possess an unobstructed central lumen to accommodate the translocation stream. CCs, on the other hand, are densely cytoplasmic, sparsely vacuolated, and have a particularly high concentration of mitochondria (Oparka and Turgeon, 1999). Because the SE is enucleate, it is generally held that the CC provides the genetic and metabolic processes necessary for SE maintenance and phloem loading (van Bel and Knoblauch, 2000).

The two cells are symplastically connected by distinct plasmodesmata characterized by multiple branches on the CC side and a single branch on the SE side that have been referred to as plasmodesmata pore units (PPUs). The presence of proteins (Fisher et al., 1992; Kühn et al., 1997; Marentes and Grusak, 1998) and nucleic acids (Xoconostle-Cazares et al., 1999; Kim et al., 2001) in the translocation stream demonstrates that the PPUs can accommodate the

\footnotetext{
${ }^{1}$ This work was supported by the U.S. Department of Agriculture/Cooperative State Research, Education, and Extension Service/National Research Initiative Competitive Grants Program (proposal no. 2001-35318-10893 to R.T.).

* Corresponding author; e-mail bga2@cornell.edu; fax 607-2555407.

Article, publication date, and citation information can be found at www.plantphysiol.org/cgi/doi/10.1104/pp.012054.
}

passage of solutes as large as macromolecules. Although the mechanism of solute trafficking is not well established, the movement of foreign proteins (Imlau et al., 1999; Oparka et al., 1999) and dextrans (Kempers and van Bel, 1997) between SEs and CCs argues that PPUs are open to the passive transfer of molecules as large as small proteins (i.e. green fluorescent protein, $27 \mathrm{kD}$; fluorescent dextrans $>10 \mathrm{kD}$ ).

Plasmodesmata that are open to the passive transfer of larger molecules would also be open to passage of smaller compounds, such that a great deal of solute flux between CCs and SEs is expected (Turgeon, 1995). Plasmolysis of SEs and CCs at approximately the same concentration of external solute (Geiger et al., 1973) suggests that PPUs equilibrate the solute concentrations between the two cells. Furthermore, fluorescent dyes approximately the size of Suc delivered to SEs of the stem are readily taken up by CCs all along the transport path (Kempers et al., 1993; Kempers and van Bel, 1997; Fujimaki et al., 2000). These observations raise an intriguing and important question: What is the fate of small solutes in the cytoplasm of CCs? The implication is that a full range of metabolites within CCs are continually lost to the translocation stream and require constant replacement. In the minor veins, where loading occurs, solute loss would be particularly acute because it is here that relatively pure water enters the SECCC from the xylem to carry off loaded photoassimilates. Extensive experimentation by Heldt and colleagues demonstrated that phloem sap contains organic and inor- 
ganic substances at concentrations similar to those in the cytosolic fraction of surrounding cells (Lohaus et al., 1995, and refs. therein).

However, the disaccharide galactinol [O- $\alpha-D$ galactopyranosyl-(1 $\rightarrow 1)$-myo-inositol], which functions as the galactosyl donor in the synthesis of the raffinose family of oligosaccharides (RFOs; Keller and Pharr, 1996), demonstrates inefficient translocation from mature leaves (Turgeon, 1996). This is despite having characteristics of an ideal transport sugar: (a) it is a nonreducing sugar with the same molecular mass as Suc; (b) in many species, significant quantities are present in the minor veins $(46 \mathrm{~mm}$ in the case of Cucumis melo; Haritatos et al., 1996); and (c) galactinol is thought to be predominantly in the cytosol along with Suc, raffinose, and stachyose (Bachmann and Keller, 1995) and the enzymes responsible for their synthesis (Holthaus and Schmitz, 1991; Beebe and Turgeon, 1992). The finding that galactinol is not efficiently translocated, therefore, is contrary to expectation if solutes originating in CCs enter the translocation stream and are transported through the phloem nonselectively. It implies that either solute transfer between CCs and SEs is somehow regulated, or that solutes not intended for longdistance transport are removed from the phloem sap.

To investigate the mechanisms controlling solute transfer between CCs and SEs and transport via the translocation stream, the distribution of traceable solutes originating in the CCs of Coleus blumei Benth. and transgenic tobacco (Nicotiana tabacum) were analyzed. The traceable solutes in C. blumei were Suc, galactinol, raffinose, and stachyose, photosynthetically labeled with ${ }^{14} \mathrm{CO}_{2}$. In transgenic tobacco, the traceable solutes were galactinol and octopine, an ionized solute with no net charge derived from Arg and pyruvate. These exotic compounds were synthesized in the CCs of mature leaves by expressing the corresponding biosynthetic genes, galactinol synthase and octopine synthase, respectively, from a minor vein-specific promoter. By this approach, intracellular delivery of the tracers was achieved in an entirely noninvasive manner.

The distribution patterns of the assayed solutes indicate unregulated movement between CCs and SEs, and consequently, unregulated entry into the translocation stream. However, substantial quantities of galactinol and octopine accumulated in nonphloem compartments, indicating poor retention within the phloem symplast. Conversely, Suc, raffinose, and especially stachyose synthesized in the minor veins of $C$. blumei moved through the phloem network with limited dilution, indicative of improved retention and efficient transport out of the leaf. We present a model in which small solutes in the CCs enter SEs and the translocation stream by an unregulated mechanism-most likely by diffusion through the PPU—but long-distance transport is governed by mechanisms of retention and/or reclamation along the transport pathway.

\section{RESULTS}

\section{Galactinol and RFO Transport in C. blumei}

C. blumei Benth. and members of the Cucurbitaceae utilize RFOs as transport sugars and are model plants for symplastic phloem loading by the polymer trap mechanism (Turgeon, 1996). According to the polymer trap model, Suc synthesized in mesophyll cells enters the intermediary cells (specialized CCs in minor veins) by diffusion through specialized, highly branched plasmodesmata. Inside the intermediary cells, Suc and galactinol are used in the synthesis of RFOs. These sugars are proposed to be too large to diffuse back toward the mesophyll and accumulate to concentrations high enough to drive bulk flow (Turgeon, 1996). In the leaves of both C. blumei and the Cucurbitaceae, the minor veins are the primary, if not the sole, sites for galactinol and RFO synthesis (Beebe and Turgeon, 1992; Turgeon and Gowan, 1992; Haritatos et al., 2000).

The distribution of ${ }^{14} \mathrm{C}$-labeled saccharides in $C$. blumei was assessed to determine the transport characteristics of each compound. Sugars were resolved by thin-layer chromatography (TLC) in two dimensions, and the amount of each sugar present was estimated by staining and autoradiography. A representative experiment was also quantified for absolute sugar concentrations and ${ }^{14} \mathrm{C}$ incorporation by HPLC (Table I): The specific activity of each sugar reflects the amount synthesized from photosynthetically fixed ${ }^{14} \mathrm{CO}_{2}$ relative to that present at the time of

\begin{tabular}{|c|c|c|c|c|c|c|}
\hline \multirow[b]{2}{*}{ Saccharide } & \multicolumn{3}{|c|}{ C. blumei Lamina } & \multicolumn{3}{|c|}{ C. blumei Petiole } \\
\hline & $\begin{array}{c}\mu \mathrm{g} g \text { fresh } w^{-1} \\
\times 10^{3}\end{array}$ & $\begin{array}{c}\mathrm{dpm} \mu \mathrm{g}^{-1} \\
\times 10^{3}\end{array}$ & $\%$ incorporation & $\begin{array}{c}\mu \mathrm{g} g \text { fresh } \mathrm{wt}^{-1} \\
\times 10^{3}\end{array}$ & $\begin{array}{c}\mathrm{dpm} \mu \mathrm{g}^{-1} \\
\times 10^{3}\end{array}$ & $\%$ incorporation \\
\hline Stachyose & 0.238 & 3.38 & 34.0 & 0.144 & 3.04 & 80.5 \\
\hline Raffinose & 0.127 & 3.14 & 17.9 & 0.060 & 1.12 & 12.5 \\
\hline Suc & 0.673 & 0.999 & 28.4 & 0.064 & 0.525 & 6.14 \\
\hline Galactinol & 0.310 & 0.496 & 6.51 & 0.327 & 0.007 & 0.44 \\
\hline Glc & 0.972 & 0.216 & 8.87 & 0.101 & 0.024 & 0.44 \\
\hline Fru & 0.846 & 0.117 & 4.19 & 0.061 & 0.000 & 0.00 \\
\hline Inositol & 0.018 & 1.41 & 1.10 & 0.008 & 0.000 & 0.00 \\
\hline
\end{tabular}


labeling and that synthesized from unlabeled carbohydrate reserves.

In the C. blumei lamina, Suc was the most prevalent nonreducing saccharide (Table I). Although hexoses were identified in greater quantities, they are not generally considered transport sugars, and their low specific activities imply they are part of, or derived from, long-term carbohydrate storage pools. The predominance of Suc was expected, because Suc is the primary product of photosynthesis in both RFO- and Suc-translocating plants.

The next most abundant sugar was galactinol (Table I). Although galactinol is synthesized in the minor veins, it is present in mesophyll cells of C. melo at concentrations as high as one-quarter that of Suc (Haritatos et al., 1996) and may show a similar distribution in the lamina of $C$. blumei. The relatively low specific activity of galactinol supports this premise because it suggests that a substantial, unlabeled pool was present.

Stachyose and raffinose were the next most abundant sugars in the leaf, respectively, and stachyose incorporated the highest percentage of ${ }^{14} \mathrm{C}$, showing that it is the major end product of photosynthesis in C. blumei (Table I). Similar to galactinol, raffinose and stachyose are synthesized in the minor veins, but unlike galactinol, are not found in flanking tissues (Haritatos et al., 1996). The high specific activity of each indicates that the unlabeled pools were small relative to those of Suc and galactinol and indicates minimal accumulation in the leaf. The finding that the products raffinose and stachyose had higher specific activities than the precursors Suc and galactinol is particularly significant because it implies that for one, and possibly both, a highly labeled pool participates in RFO synthesis and a second pool with lower specific activity is excluded, presumably by some form of physical separation.

Labeled sugars in the petiole represent those that originated in the lamina and exited the leaf via the translocation stream during the course of the labeling experiment. Comparing the specific activity of each sugar between the petiole and the lamina provides information on the relative contribution of translocated (labeled) and local (unlabeled) pools to the total amount present. With the exception of stachyose, all analyzed sugars demonstrated a prominent reduction in specific activity, indicating the petiole had an unlabeled pool of each. Galactinol in particular demonstrated a substantial drop in specific activity, and accounted for a negligible percentage of translocated ${ }^{14} \mathrm{C}$. The specific activity of stachyose, however, was only modestly decreased, indicating that the stachyose in the petiole was derived directly from the translocation stream, with little dilution from a local, unlabeled pool. That stachyose is the predominant transport sugar in this species is emphasized by the high percentage of ${ }^{14} \mathrm{C}$ incorporated into stachyose recovered from the petiole (Table I).

\section{Galactinol Transport in Transgenic Tobacco}

To further assess the transport properties of galactinol synthesized in minor veins, a genomic clone of galactinol synthase from C. melo was introduced as a transgene to tobacco. Tobacco was considered a good heterologous host for studying galactinol transport because it does not synthesize detectable quantities of galactinol or RFOs in the leaf (Haritatos et al., 2000). In addition, tobacco loads Suc from the apoplast by cotransport with protons (Giaquinta, 1983; Burkle et al., 1998) and utilizes Suc exclusively as a transport sugar.

Soluble sugars in mature leaves were assayed from 24 hygromycin-resistant plants by one-dimensional TLC and detection with vanillin staining (not shown). Galactinol was prevalent and accumulated to roughly equivalent amounts in the majority of these independently transformed plants. This result demonstrates that the substrates for galactinol synthesis, myo-inositol and UDP-Gal, are readily available despite being maintained at relatively low steady-state concentrations in plant cells (Keller and Pharr, 1996; Loewus and Murthy, 2000). Furthermore, the consistently high concentration among independently transformed plants implies that galactinol is stable in tobacco. Despite this accumulation, the plants did not demonstrate obvious growth defects to indicate that this altered carbon partitioning is deleterious to growth or development (not shown).

$\mathrm{A} \mathrm{T}_{2}$ progeny plant of a transformant displaying typical galactinol accumulation patterns was labeled

\begin{tabular}{|c|c|c|c|c|c|c|c|}
\hline \multirow[b]{2}{*}{ Saccharide } & \multicolumn{3}{|c|}{ Transgenic Tobacco Lamina } & \multicolumn{3}{|c|}{ Transgenic Tobacco Midrib } & \multirow{2}{*}{$\begin{array}{c}\text { Transgenic Tobacco Apoplast } \\
\mu \mathrm{g} \mathrm{mL}-1 \mathrm{AF} \times 10^{3}\end{array}$} \\
\hline & $\begin{array}{c}\mu \mathrm{gg} \text { fresh } \mathrm{wt}^{-1} \\
\times 10^{3}\end{array}$ & $\mathrm{dpm} \mu \mathrm{g}^{-1}$ & $\%$ incorporation & $\begin{array}{c}\mu \mathrm{gg} \text { fresh wt }{ }^{-1} \\
\times 10^{3}\end{array}$ & $\mathrm{dpm} \mu \mathrm{g}^{-1}$ & $\%$ incorporation & \\
\hline Raffinose & 0.181 & 0.00 & 0.00 & 0.074 & 70.0 & 4.98 & 0.00 \\
\hline Suc & 1.53 & 400 & 62.0 & 1.15 & 76.4 & 84.3 & 0.564 \\
\hline Galactinol & 11.5 & 21.0 & 24.2 & 3.39 & 1.36 & 4.41 & 3.34 \\
\hline Inositol & 0.170 & 48.0 & 0.80 & 0.017 & 37.3 & 0.616 & 0.030 \\
\hline
\end{tabular}

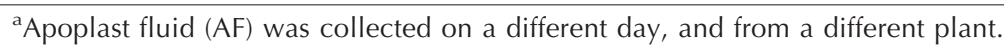



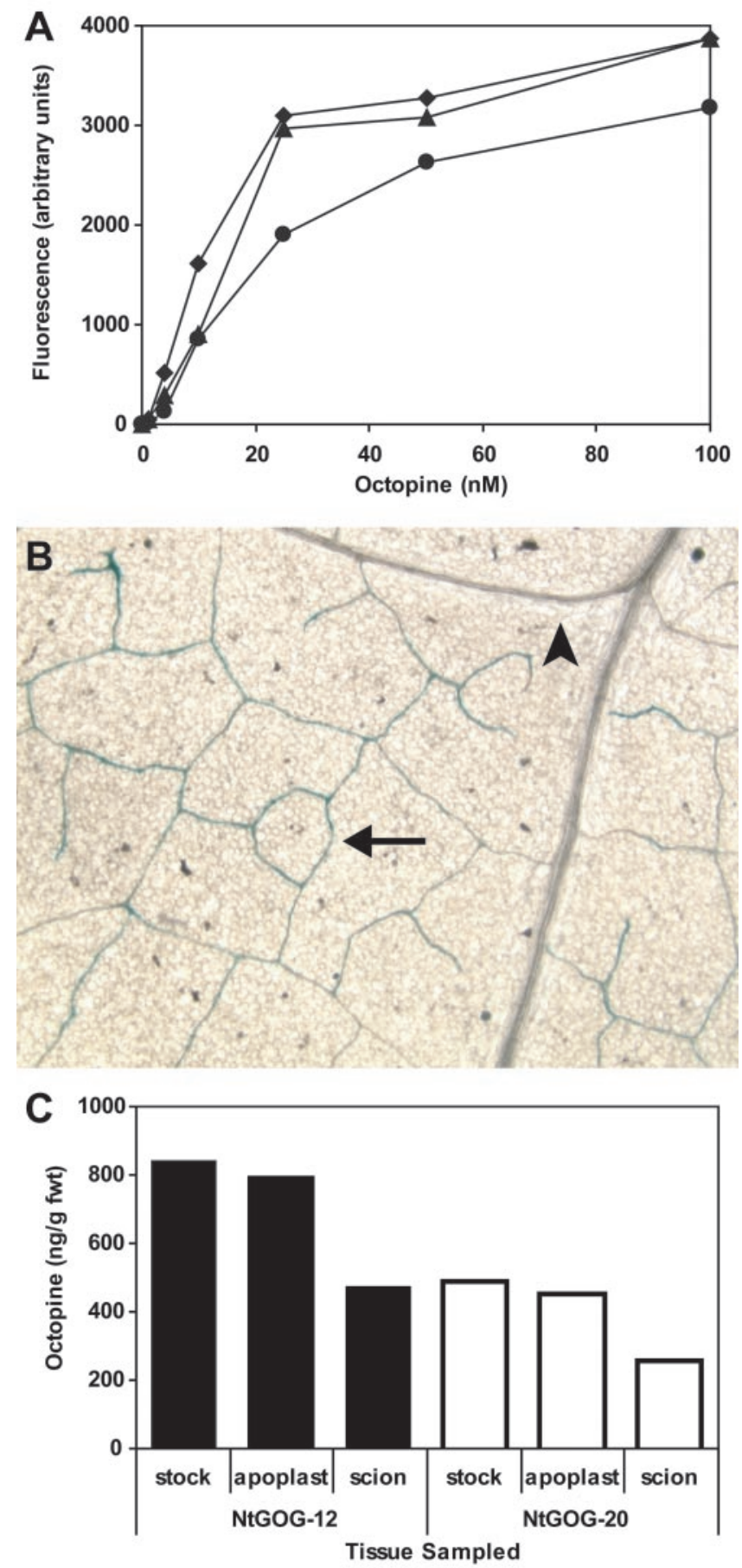

Figure 1. Quantitative bioassay for octopine, and distribution patterns throughout transgenic tobacco. A, Representative standard curve for detecting octopine in the growth medium of Agrobacterium tumefaciens strain KYC16 in the absence of wild-type plant extract $(\downarrow)$, with the addition of extract from $10 \mathrm{mg}$ fresh weight wild-type leaf tissue $(\boldsymbol{\Lambda})$, and with the addition of extract from $50 \mathrm{mg}$ fresh weight wild-type leaf tissue (-) B, Leaf section of NtGOG-12 tobacco plant stained with the $\beta$-glucuronidase (GUS) substrate 5 -bromo-4-chloro-3-indolyl- $\beta$-D-GlcUA (X-GlcA) and cleared of chlorophyll. Staining in a minor vein is indicated with an arrow, whereas an arrowhead indicates the absence of staining in a larger vein. C, Representative distribution of octopine in grafted tobacco plants. Stock, Mature leaf tissue from the indicated transgenic tobacco with ${ }^{14} \mathrm{CO}_{2}$, and soluble sugars were analyzed as described above for C. blumei (Table II). In the lamina of the mature leaf, the Suc concentration was $1.53 \times$ $10^{3} \mu \mathrm{g} g$ fresh weight ${ }^{-1}$ and the specific activity was $400 \mathrm{dpm} \mu \mathrm{g}^{-1}$. Galactinol accounted for nearly onequarter of the incorporated ${ }^{14} \mathrm{C}$, indicating substantial synthesis during the labeling period, and accumulated to $11.5 \times 10^{3} \mu \mathrm{g}$ g fresh weight ${ }^{-1}$, exceeding the Suc concentration nearly 8 -fold. The specific activity, however, was only $24.0 \mathrm{dpm} \mu \mathrm{g}^{-1}$, supporting the premise that galactinol is stable and long lived in tobacco. Unexpectedly, raffinose was present in the lamina in minor quantities. Raffinose incorporated negligible label when assayed by HPLC with radioisotope detection; however, it was detectable by autoradiography after two-dimensional TLC (not shown).

The predominant transport (labeled) sugar identified by assaying the midrib was Suc (Table II). The specific activity of Suc in the midrib was less than that in the lamina and points to a local pool in addition to that derived from the translocation stream. The concentration of galactinol in the midrib was $3.39 \times 10^{3} \mu \mathrm{g} g$ fresh weight ${ }^{-1}$ : less than that observed in the lamina, but still exceeding the total amount of Suc in the midrib $\left(1.15 \times 10^{3} \mu \mathrm{g}\right.$ g fresh weight $^{-1}$ ) by nearly 3 -fold. The specific activity of galactinol was very low, indicating a large, and presumably long-lived, local pool. This finding was not anticipated because the galactinol synthase promoter drives gene expression in the minor veins of tobacco (Haritatos et al., 2000) but paralleled the results observed in the petiole of $C$. blumei. Raffinose was also present in the petiole in minor quantities, as observed in the lamina.

The distribution of galactinol was further assessed by measuring the saccharide content of the apoplastic fluid in mature leaves (Table II). The concentration of galactinol in the apoplast exceeded that of Suc nearly 6 -fold, demonstrating that there is substantial movement from the sites of synthesis to alternative compartments and tissues. Similarly, galactinol was identified by two-dimensional TLC in wild-type scions grafted to galactinol-synthesizing stock plants (not shown).

\section{Octopine Transport in Transgenic Tobacco}

Galactinol metabolism is ubiquitous among plant species because RFO synthesis occurs in maturing seeds, frequently under drought or cold stress, and as a prominent transport compound in some plants (Keller and Pharr, 1996). These endogenous biochem-

\footnotetext{
plant; solid bars, NtGOG-12; and hollow bars, NtGOG-20. Apoplast, Apoplast washing fluid collected from a mature leaf sample of the indicated transgenic plant, corrected for dilution, and assuming a specific gravity of one. Scion, Apical bud tissue of a wild-type scion grafted to the indicated transgenic stock plant.
} 
ical pathways can compromise the analysis of galactinol transport, as evidenced by the unexpected utilization of galactinol for the production of raffinose in the tobacco leaf. To overcome these limitations, octopine was selected as an alternative tracer for establishing the transport fate of small solutes in the CCs. Octopine metabolism does not naturally occur in plants but is derived from readily available precursors, Arg and pyruvate, in a reaction catalyzed by a single enzyme, octopine synthase (Dessaux and Petit, 1994). Therefore, octopine is readily synthesized and stable in plant cells expressing the octopine synthase gene but, as a foreign compound, should not be recognized by specific transporters or carriers.

Although several chromatographic assays exist for identifying octopine in plant extracts, they are laborious, insufficiently sensitive, and can be misleading because they detect many endogenous plant compounds in addition to octopine (Maille, 1983; Dessaux and Petit, 1994). As a consequence, we developed a novel bioassay based on the octopine-inducible, octopine catabolism pathway of octopine-type A. tumefaciens strains, encoded on the occ operon (Cho et al., 1996). This bioassay utilizes an A. tumefaciens strain with the gusA7 gene inserted into the occ operon such that it becomes induced when the strain is grown in the presence of octopine (Fig. 1A, see "Materials and Methods" for details).

To synthesize octopine specifically in CCs of transgenic tobacco, a fusion gene was created between ocs and uidA (encoding the GUS protein), and placed downstream of the CmGAS1 promoter. Octopine synthase was fused to GUS for two reasons. First, the predicted molecular mass for octopine synthase is $38.8 \mathrm{kD}$, and the size exclusion limit for PPU between CCs and SEs may be as high as $60 \mathrm{kD}$ for globular proteins (Kempers and van Bel, 1997). However, the OCS-GUS fusion protein has a predicted mass of 110 $\mathrm{kD}$ and should be restricted to the CCs. Second, histochemical staining of tissues with the GUS substrate, X-GlcA, enabled precise localization of octopine synthesis.

Thirty-six tobacco plants, independently transformed with pGAS-OCS/GUS, were analyzed for GUS activity and the presence of octopine in mature leaves. Two plants demonstrating the highest levels of both indicators were used in further studies. These plants were designated NtGOG-12 and NtGOG-20.

Long-distance transport of octopine was assayed in grafting experiments with wild-type tobacco as scions and plants segregating for the presence of the fusion gene as stock. As expected, the OCS-GUS fusion protein was restricted to the minor veins of transgenic stock plants as indicated by histochemical staining for GUS activity (Fig. 1B). Fluorescent assays on plant extracts with the 4-methylumbelliferyl $\beta$-Dglucuronide hydrate substrate revealed GUS activity only in mature source leaves of the stock plants. GUS activity was not detected above background values in stock sink tissues or in any tissues of the wild-type scion (not shown). These results demonstrate that the OCS-GUS enzyme does not enter the translocation stream.

However, the product of this enzyme, octopine, was detected throughout the plant (Fig. 1C). Octopine was detected in the sink tissues of the scion, indicating that octopine synthesized in the CCs can enter the SE and move long distances via the translocation stream. Furthermore, octopine was detected in apoplastic fluids when collected with approaches demonstrated to minimize cellular damage (Husted and Schjoerring, 1995; Lohaus et al., 2001). This result demonstrates that octopine produced in the symplast enters the apoplast. It is particularly noteworthy that the levels of octopine detected in the scion and source-leaf apoplast are very close to those detected in the whole-leaf samples (Fig. 1C). This finding implies that octopine becomes distributed more or less equally throughout the plant despite its highly localized synthesis.

Because octopine is able to move from the symplast to the apoplast, it is possible that upon reaching the stem, acropetal transport into the wild-type scion may have occurred in the transpiration stream of the xylem, rather than the translocation stream of the phloem. To establish phloem transport, reciprocal grafts were made such that wild-type plants formed the rootstock, and transgenic plants were used as scions. Two weeks after grafting, octopine was readily detected in roots, demonstrating phloem transport (not shown).

The presence of octopine in the apoplast also raised the possibility that entry into the SEs may have occurred by transmembrane transport rather than through the PPUs connecting the CCs and SEs. To test this possibility, $6.15 \mu \mathrm{g}$ of octopine was delivered to the apoplast of a mature leaf, along with $3.16 \times 10^{6}$ $\mathrm{dpm}$ of ${ }^{14} \mathrm{C}$-labeled Suc as a second tracer (both at 100 $\mu \mathrm{M})$. After $24 \mathrm{~h}, 0.54 \%$ of the ${ }^{14} \mathrm{C}$ was extracted from $100 \mathrm{mg}$ fresh weight of tissue at the vegetative apex. If octopine in the apoplast entered and moved through the translocation stream with similar efficiency, $33 \mathrm{ng}$ ( $330 \mathrm{ng} g$ fresh weight ${ }^{-1}$ ) would have been anticipated in the same tissue. However, no octopine was detected under assay conditions expected to exceed the detection limit 50-fold (not shown). This finding suggests that in the transgenic plants, octopine that leaks into the apoplast contributes little to the transport pool and supports the premise that octopine was transported primarily through the symplast.

\section{DISCUSSION}

Translocation through the phloem is the result of a pressure gradient between sink tissues (low pressure) and source tissues (high pressure). In mature leaves, high osmotic pressure is generated by the 
active accumulation of photoassimilate into the SECCC of minor veins, and is referred to as phloem loading. Thus, minor veins-positioned at the inception of the translocation stream-are located where relatively pure water enters from the xylem to carry away dissolved sugar and other compounds. This persistent dilution of the SECCC contents would seem metabolically costly if solute transfer between the cytoplasm-rich CCs and degenerate SEs is indiscriminate. However, the conspicuous absence of certain metabolites from the translocation stream implies that the content of the phloem sap is to some extent regulated. To study solute flux within the SECCC and to surrounding tissues in relation to the translocation stream, we analyzed the dispersion patterns of traceable solutes synthesized in the CCs of minor veins in C. blumei and transgenic tobacco.

\section{Octopine Distribution}

One of these traceable solutes was octopine. Octopine is a small molecule (246.3 D) derived from Arg and pyruvate, which is ionized but has no net charge at physiological $\mathrm{pH}$. The enzyme responsible for octopine synthesis, octopine synthase, was expressed as a fusion with GUS in transgenic tobacco. The resulting enzyme has a predicted molecular mass of 110 $\mathrm{kD}$. Although molecular mass does not directly reflect the hydrodynamic radius, we reasoned that a protein of this size would exceed the size exclusion limits of PPUs and be restricted to the CCs in which it was translated. Importantly, the GUS fusion allowed precise protein localization by histochemical staining, and consequently, precise identification of the sites of octopine synthesis. The fusion protein, and thus octopine synthesis, localized to the minor veins and was excluded from heterotrophic tissues (Fig. 1B).

Despite this localized synthesis, octopine was detected in all tissues examined: source leaves, apoplastic fluids, and heterotrophic tissues, including the aerial sinks of wild-type scions (Fig. 1C) and wildtype roots in reciprocal grafts (not shown). These results demonstrate that octopine is transported from the site of synthesis in CCs of minor veins to SEs, and subsequently long distance to sink tissues via the translocation stream. However, octopine in the apoplast suggests that in addition to translocation, dispersal throughout the leaf may occur in the transpiration stream.

Two paths for entry into the SEs are theoretically possible. The most probable route from CC to SE is symplastically through the PPUs that connect the two cell types. The alternative route is from CC to the apoplast, followed by uptake into the SE. However, exogenous delivery of octopine to the apoplast did not result in detectable transport, suggesting that octopine that leaks into the apoplast is removed from the transport pool. This finding is expected for a foreign molecule that is not recognized by a transporter, because the proximity and relative purity of water in the xylem draws solutes leaching from the phloem into the transpiration stream and effectively concentrates them in the leaf (Hsu and Kleier, 1996). Therefore, the best explanation for long-distance transport of octopine is efficient retention within the phloem symplast resulting from the plasma membrane's relative impermeability to ionized molecules. That is, octopine produced in the CCs enters the SEs and the translocation stream through the PPUs and is efficiently retained within the translocation stream to the sink tissues. Notwithstanding, gradual transmembrane leakage undoubtedly occurs, both from the sites of synthesis in the CCs and along the phloem network, resulting in the observed accumulation of octopine in the apoplast (Fig. 2).

\section{Galactinol Distribution}

The second traceable compound synthesized in the CCs of minor veins in transgenic tobacco was galactinol. In C. blumei and the Cucurbitaceae, galactinol is synthesized in the intermediary cells (specialized CCs) of minor veins as a precursor for the synthesis of RFO (Beebe and Turgeon, 1992; Turgeon and Gowan, 1992). In wild-type tobacco, galactinol is not detected in leaves (Haritatos et al., 2000), but plants transformed with the CmGAS1 genomic clone from C. melo have galactinol concentrations in mature leaf tissues that dramatically exceed those of Suc (Table II).

The distribution of galactinol in transgenic tobacco plants paralleled that of octopine in that it was identified in high concentrations in apoplastic fluids and heterotrophic tissues. However, in labeling experiments, the majority of labeled galactinol did not reach the petiole of either $C$. blumei or the midrib of transgenic tobacco (Tables I and II). Our interpretation of these results is that galactinol behaves similarly to octopine, but as an unionized compound, it is retained less well within the phloem symplast. Specifically, galactinol produced in the minor veins is distributed to surrounding tissues and compartments by passive efflux, including symplastic entry into the SEs through the PPUs and entry into the apoplast. The proportion of galactinol that enters the translocation stream is then subject to further passive efflux as it moves along the path phloem (Fig. 2).

This model for inefficient translocation of galactinol being the result of poor retention within the phloem symplast is well supported by extensive studies on the translocation of Suc. Transmembrane Suc efflux, or leakage, occurs both in the minor veins at the sites of loading and along the path phloem on the way to regions of demand (Turgeon, 1984; van Bel, 1993; Thorpe and Minchin, 1996). In the latter case, Suc loss along the path phloem was measured at $6 \%$ per centimeter. Therefore, efflux of Suc from the phloem symplast is substantial; however, Suc 


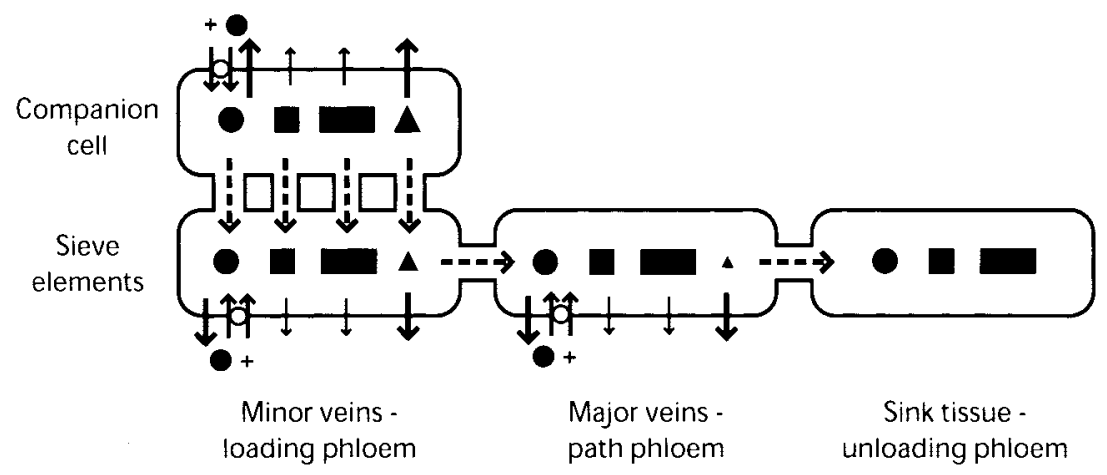

Figure 2. Schematic representation of the distribution of solutes originating in the CC of minor veins. Net diffusion of small solutes in the CCs is in the direction of the SEs and the translocation stream (dashed arrows). Solutes may also diffuse across membranes as a function of their permeability coefficients. The relative rates of transmembrane diffusion are represented by the size of the solid arrows. The relative amounts of each solute remaining in the symplast along the phloem pathway are represented by the size of the respective symbols. Octopine (squares) is ionized, and is efficiently retained in and translocated through the phloem. Gradual leakage and accumulation in the apoplast does occur. Suc (solid circles) is pumped into the SECCC with protons $(+)$ by Suc transporters (white circles). Suc leaks from the phloem symplast at appreciable rates but is retrieved by transporters situated throughout the phloem network. Galactinol (triangles) also leaks at appreciable rates but is not retrieved, and it is eventually depleted from the translocation stream. The larger saccharide stachyose (rectangles) leaks minimally and is efficiently transported to sink tissues. Although not represented, diffusion of some solutes from the SECCC to mesophyll cells through plasmodesmata may also occur. For simplicity, CCs in the path and sink tissues are not represented.

transporters in the minor veins and along the path phloem mediate loading and retrieval from the apoplast and ensure efficient transport (Fig. 2; van Bel, 1993; Thorpe and Minchin, 1996).

Although galactinol is present in the apoplast, transmembrane leakage is not the only possible mode of distribution to tissues surrounding the SECCC. In the minor veins of all species studied to date, plasmodesmata are present between the SECCC and surrounding tissues (Turgeon et al., 2001). Therefore, a symplastic route from the sites of synthesis in the CCs to surrounding cells, including the mesophyll, is possible. In species that load photoassimilate symplastically by the polymer trap mechanism, these plasmodesmata are extremely numerous and appear to be open for the passage of Suc and galactinol (Haritatos et al., 1996; Turgeon, 1996). In species like tobacco that load from the apoplast, these plasmodesmata are less frequent but are nevertheless present.

Notwithstanding the inefficient translocation of galactinol, small quantities of labeled galactinol do reach the midrib and petiole, indicating that retention within the symplast of the phloem is sufficient for long-distance transport of small amounts. The gradual loss of galactinol along the path phloem and accumulation in surrounding tissues explains the significant presence of galactinol in tissues far removed from the sites of synthesis in minor veins.

\section{RFO Distribution}

In the leaves of wild-type tobacco, RFOs and their essential precursor galactinol are not detected (Haritatos et al., 2000), but a small amount of raffinose was present in transgenic plants that synthesized galacti- nol (Table II). The best candidate enzyme for synthesizing raffinose from galactinol is raffinose synthase; however, it is not clear why mature tobacco leaves would have this enzyme activity. The occurrence of RFOs in plants is nearly ubiquitous and is implicated in carbon transport, carbon storage, and cold and desiccation tolerance (Keller and Pharr, 1996). Thus, the activity observed in tobacco leaves may be the result of low-level expression of a seed-specific gene, or a remnant raffinose synthase from an ancestral species that utilized RFO as a transport sugar (Turgeon et al., 2001).

In C. blumei, RFOs are the predominant transport sugars and are synthesized in the minor veins of mature leaves (Turgeon and Gowan, 1992). The high specific activities of raffinose and stachyose indicate that the pool of accumulated (unlabeled) RFOs in the leaf was relatively small. The lower specific activities of the precursors, Suc and galactinol, suggest that there is a highly labeled pool of each involved in RFO synthesis and a second pool with lower activity that is excluded. It is reasonable to speculate that the pool of highly labeled precursors involved in RFO synthesis are derived from photoassimilate loaded into the phloem during the labeling experiment, whereas the second pool are those synthesized before labeling and sequestered in non-phloem compartments.

The petiole was not directly labeled by photoassimilation of ${ }^{14} \mathrm{CO}_{2}$; thus, radiolabeled sugars in the petiole were those transported out of the labeled lamina. Because stachyose, raffinose, and galactinol are synthesized in the minor veins, the unlabeled pools of each in the petiole likely entered the translocation stream before labeling and leached into surrounding tissues as they moved along the phloem 
network. With the notable exception of stachyose, the specific activities of all sugars in the petiole were markedly reduced relative to those observed in the lamina, indicating a local pool of each. Stachyose, however, demonstrated a very modest reduction in specific activity from the lamina to the petiole, suggesting that only a very small, local pool was present in the petiole, and the majority detected was in the translocation stream.

An effective mechanism for keeping the local pool of RFOs small in the lamina and petiole would be efficient transport out of the leaf. Stachyose, as a tetrasaccharide, is predicted to be too large to pass through plasmodesmata between intermediary cells and bundle sheath cells (Turgeon, 1996) and to demonstrate greater membrane retention than a disaccharide. Improved retention in the phloem symplast would bring about greater translocation efficiency and faster removal from the source leaf (Fig. 2). The supposition that larger saccharides are less membrane permeant than smaller ones is supported by comparisons of the linear-component uptake rates of different sugars into the plant symplast: Raffinose is slower than Suc, and stachyose is slower still (Bennet et al., 1986; Madore, 1990; Turgeon and Gowan, 1990).

\section{Solute Retention/Reclamation and Münch Pressure Flow}

The distribution of octopine and galactinol synthesized in the minor veins is most consistent with unregulated movement between CCs and SEs and subsequent long-distance transport in the translocation stream. However, leaching of both compounds from the phloem was significant, with galactinol leaching to a greater extent than octopine. Ernst Münch proposed that the translocation stream of the phloem is driven by a pressure differential between source and sink tissues and recognized that to maintain a pressure gradient, the conducting elements must be leakfree, or nearly so (for discussion, see van Bel, 1993; Thorpe and Minchin, 1996). Leakage of transport sugars from the SECCC would compromise the efficiency of translocation and, consequently, mechanisms to minimize solute loss have evolved. For example, Suc is lost from the phloem at appreciable rates, but Suc transporters line the path phloem for retrieval from the apoplast (van Bel, 1993; Thorpe and Minchin, 1996; Kühn et al., 1997). Even in species that load symplastically and transport primarily RFOs, Suc transporters are expected in the minor veins to retrieve leaked Suc (for discussion, see Grusak et al., 1996). Experimental evidence supporting this was recently obtained (Knop et al., 2001).

Galactinol, like Suc, is a nonreducing disaccharide that demonstrates significant efflux from the phloem symplast, but unlike Suc does not appear to be retrieved. Rather, galactinol functions as a precursor in the synthesis of raffinose, stachyose, and higher order oligosaccharides (Keller and Pharr, 1996). Based on the findings that RFOs, especially stachyose, demonstrate minimal accumulation in the leaf and petiole and that higher order oligosaccharides in general have reduced membrane permeation rates, we propose that conversion of disaccharides to tri- and tetrasaccharides is another mechanism that plants employ to maintain the integrity of the pressure gradient between source and sink tissues. Therefore, in species that transport RFOs, polymer trapping may fulfill two roles essential for efficient translocation. First, polymer trapping is required for phloem loading (Turgeon, 1995, 1996), and second, polymer trapping helps reduce solute leakage during longdistance transport.

\section{MATERIALS AND METHODS}

\section{Plasmid Construction}

All plasmid constructions were by standard procedures (Ausubel et al., 1995). Enzymes and reagents were obtained from New England Biolabs (Beverly, MA) and Invitrogen (Carlsbad, CA), and used according to the manufacturers' instructions. The Cornell BioResource Center (Ithaca, NY) performed sequencing reactions.

Plasmid pCamGAS1 was created to express the CmGAS1 galactinol synthase gene in the minor veins of transgenic tobacco (Nicotiana tabacum) plants. The CmGAS1 expression cassette corresponds to a 6-kb EcoRI fragment of Cucumis melo genomic DNA contained on pSG8E (Haritatos et al., 2000). This cassette includes the 3-kb CmGAS1 promoter region demonstrated to confer minor vein expression to heterologous species (Haritatos et al., 2000), the galactinol synthase gene including introns, and $0.5 \mathrm{~kb}$ of $3^{\prime}$-untranslated sequence. The cassette was excised from pSG8E with EcoRI, gel purified with a QIAquick Gel Extraction Kit (Qiagen, Valencia, CA), and cloned into the EcoRI site of the binary vector pCambia1301 (www.cambia.org).

The plasmid pGAS-OCS/GUS was created to express an octopine synthase gene fused in-frame to the GUS reporter gene (uidA) in the minor veins of transgenic plants. Initially, the CmGAS1 promoter without an ATG translation initiation codon was subcloned into pUC19 to create pUC-GUT. This was done in two steps. First, sequences -1 to $-1,078$ (relative to the CmGAS1 start codon) were amplified in a $50-\mu \mathrm{L}$ PCR reaction with the forward oligonucleotide TCAAGGATCCGCCACCACAATCAGAC, the reverse oligonucleotide CGTTGCTGCAGGGTACCTATTGACTTTGGTGCTTTTG, a pSG8E-derived plasmid as template, and 2.5 units of AmpliTaq DNA polymerase (Applied Biosystems, Foster City, CA). The cycling parameters were melting at $94^{\circ} \mathrm{C}$ for $30 \mathrm{~s}$, annealing at $55^{\circ} \mathrm{C}$ for $60 \mathrm{~s}$, and elongation at $72^{\circ} \mathrm{C}$ for $120 \mathrm{~s}$, repeated for 30 cycles. The resulting PCR product was digested with BamHI and KpnI (recognition sites underlined in oligonucleotide sequences), gel purified, and inserted into the same sites of pUC19. Second, a BamHI/PstI promoter fragment corresponding to CmGAS1 sequences $-1,078$ to $-3,086$ was subcloned into this vector from pSG3K101 (Haritatos et al., 2000), thereby recreating the CmGAS1 promoter with a convenient KpnI recognition site in place of CmGAS1 coding sequences.

An octopine synthase (ocs) gene fragment lacking a stop codon was synthesized by PCR. The source of this gene was a pBR325 plasmid containing the ocs gene from pTiA6 (Escherichia coli strain E22; a gift from Stanton Gelvin, Purdue University, West Lafayette, IN). The forward oligonucleotide sequence was TATCGAGGTACCGATGGCTAAAGTGGCAATTTTGG, and the reverse oligonucleotide sequence was CTTAAGAGCTCCCCGGGGAACTCCATTGAGAGCCCTGA. The cycling parameters were as above, except that the annealing step was for $30 \mathrm{~s}$ and initiated at $65^{\circ} \mathrm{C}$, dropped $0.7^{\circ} \mathrm{C}$ each cycle for 11 cycle, and continued at $55^{\circ} \mathrm{C}$ for an additional 25 cycles, and that the extension step was for $90 \mathrm{~s}$. This gene fragment was subcloned into a pGEM3zf+ (Promega, Madison, WI) derivative as a KpnI/SacI (recognition sites underlined in the oligonucleotides) fragment, and sequenced. The uidA gene was isolated from pBi101.2 (Jefferson et al., 1987) as an XmaI/SacI fragment and cloned downstream of the ocs sequence (XmaI site underlined in the ocs reverse oligonucleotide) to create an in-frame fusion. The fusion gene 
was isolated as a $\mathrm{KpnI} / \mathrm{SacI}$ fragment and subcloned into the same sites of pUC-GUT, placing the fusion gene downstream of the CmGAS1 promoter. The CmGAS1 promoter-ocs-uidA cassette was then subcloned into pGPTV-kan (Becker et al., 1992) with $\mathrm{XbaI}$ and SacI to create pGAS-OCS/GUS.

\section{Plant Material}

Variegated Coleus blumei Benth. was grown as previously described (Turgeon and Gowan, 1992). Tobacco cv petite Havana SR1 was a gift from Mikhail Nasrallah (Cornell University, Ithaca, NY) and grown as described by Haritatos et al. (2000). The binary vectors pGAS-OCS/GUS and pCamGAS were introduced to Agrobacterium tumefaciens strain GV3101, and generation of transgenic plants was also as described (Haritatos et al., 2000) Plantlets that formed roots in the presence of $100 \mathrm{mg} \mathrm{L}^{-1}$ kanamycin sulfate (pGAS-OCS/GUS) or $15 \mathrm{mg} \mathrm{L}^{-1}$ hygromycin sulfate (pCamGAS1) were transferred to 12.5-cm pots containing Metro-Mix 360 (Scotts, Marysville, $\mathrm{OH})$. Histochemical staining for GUS enzyme activity with X-GlcA was as described by Haritatos et al. (2000).

For grafting experiments, transgenic stock plants were grown to a height of approximately $20 \mathrm{~cm}$ and had at least four fully mature leaves $15 \mathrm{~cm}$ or greater in length. Approximately $2.5 \mathrm{~cm}$ of the shoot apex was removed by a horizontal cut with a razor blade. For the scion, a similarly sized shoot apex was cut from a wild-type plant and secured to the stock plant with a sewing pin. Grafted plants were kept at $100 \%$ relative humidity for several days until the graft healed, acclimatized, and returned to the controlled environment chamber. Grafted plants were grown for another 2 weeks before taking samples for analysis.

Apoplastic fluid was collected by the infiltration centrifugation technique. Excised leaf samples that excluded the midrib were submersed in deionized water and brought to $15 \mathrm{kPa}$ in a vacuum chamber. Water entered the samples upon release of the vacuum. The samples were blotted dry and placed on parafilm. The leaf and parafilm were wrapped together around a 1-mL syringe barrel and placed inside a $5-\mathrm{mL}$ syringe barrel that was in turn placed in a 15-mL Falcon tube. Apoplastic fluid was collected by centrifugation in a swinging bucket rotor for $10 \mathrm{~min}$ at $250 \mathrm{~g}$. To minimize resistance to elution, the secondary veins of the leaf samples were oriented parallel to the long axis of the Falcon tube. Dilution of the apoplastic fluid was conservatively estimated to be 4-fold (Husted and Schjoerring, 1995; Lohaus et al., 2001).

\section{Octopine Assays}

A quantitative bioassay based on the octopine-inducible, octopineutilization pathway of A. tumefaciens was established for measuring octopine concentration in plant extracts. A. tumefaciens KYC16 is an R10-derived strain with a promoterless Tn5-gusA7 construct inserted into the octopine oxidase gene (ooxA) of the octopine utilization operon (occ; Cho et al., 1996). The occ operon, and, consequently, the gusA7 gene, encoding GUS, is induced by the presence of octopine in the growth medium. Furthermore, because the insertion disrupts the ooxA gene, KYC16 cells are unable to degrade the octopine inducer, ensuring that the cells remain in the induced state. Standard assay conditions were established. "Pre-induced" cells were prepared as described by Cangelosi et al. (1991), and $10 \mu \mathrm{L}$ was incubated with $1 \mathrm{~mL}$ of AB media (Cangelosi et al., 1991) supplemented with commercial octopine (Sigma, St. Louis) or plant extracts equivalent to $5 \mathrm{mg}$ fresh weight tissue as required. Cultures were incubated for $7 \mathrm{~h}$ at $30^{\circ} \mathrm{C}$ with shaking at $300 \mathrm{rpm}$ to provide aeration. Culture density was determined by diluting $500 \mu \mathrm{L}$ into $3 \mathrm{~mL}$ of distilled water, and measuring the $\mathrm{OD}_{600}$. The remaining culture was frozen overnight to permeabilize the cells. To measure GUS enzyme activity in the cultures, $10 \mu \mathrm{L}$ of thawed culture was incubated for $30 \mathrm{~min}$ at $37^{\circ} \mathrm{C}$ with $100 \mu \mathrm{L}$ of prewarmed MUG assay solution (1 mM 4-methylumbelliferyl $\beta$-D-glucuronide hydrate, $50 \mathrm{~mm}$ sodium phosphate buffer [pH 7.0], $10 \mathrm{~mm} 2$-mercaptoethanol, and $0.1 \%$ [v/v] Triton X-100; Jefferson et al., 1987). Reactions were terminated by the addition of 3 $\mathrm{mL}$ of $0.2 \mathrm{M} \mathrm{Na}_{2} \mathrm{CO}_{3}$, and fluorescence was measured in an FP-750 spectrofluorometer (Jasco, Easton, MD). Fluorescence was standardized against culture density.

To quantify octopine levels in plant extracts, fluorescence resulting from octopine in leaf extracts was compared with fluorescence resulting from commercial octopine standards. To extract octopine from transgenic leaf tissue, samples that excluded the midrib were crushed in an equal volume of water $(\mathrm{w} / \mathrm{v})$ and incubated for $15 \mathrm{~min}$ at $95^{\circ} \mathrm{C}$. The water was then removed to a fresh tube, and the extraction was repeated three times (Dessaux and Petit, 1994).

Induction of gusA7 was negligible in the absence of octopine but was readily detectable at concentrations as low as $1 \mathrm{~nm}$. Octopine concentrations from 4 to $25 \mathrm{~nm}$ gave an approximately linear increase in response, whereas cultures containing $50 \mathrm{~nm}$ octopine or greater did not demonstrate increased GUS activity, indicating saturation of the induction system (Fig. 1A). The addition of wild-type extracts from $10 \mathrm{mg}$ fresh weight leaf tissue had minimal impact on the standard curves, whereas the addition of wild-type extract from $50 \mathrm{mg}$ fresh weight leaf tissue reduced induction slightly, possibly representing modest catabolite repression (Fig. 1A). However, because extract from $5 \mathrm{mg}$ fresh weight tissue or less was used to assay our transgenics plants, this finding has no appreciable affect on our measurements. Importantly, the addition of wild-type extract alone did not result in $g u s A 7$ induction, proving that this bioassay is specific for octopine and not induced by endogenous plant compounds.

To assess octopine uptake and transport from the apoplast, $6.15 \mu \mathrm{g}$ of octopine and $3.16 \times 10^{6} \mathrm{dpm}$ of ${ }^{14} \mathrm{C}$-labeled Suc were delivered to the apoplast of a mature leaf in a solution containing $100 \mu \mathrm{M}$ of each. A pin was used to make a small hole in the lower epidermis of the leaf, over which the opening of a 1-mL syringe barrel containing the tracer solution was placed. Gentle pressure between the leaf and the syringe barrel was applied to form a seal, and the solution was then pushed into the intercellular spaces by gentle pressure on the syringe plunger. This flooded approximately $2 \mathrm{~cm}^{2}$ of the leaf, and the process was repeated until $20 \mathrm{~cm}^{2}$ of leaf area was treated. Leaf samples were collected with a $4-\mathrm{mm}$ diameter cork bore, and the total amount of solution delivered was determined by liquid scintillation counting (Turgeon and Gowan, 1992).

All source leaves except the treated one were removed, as were all immature leaves except those less than $1.5 \mathrm{~cm}$ in length at the vegetative apex. After $24 \mathrm{~h}$, the vegetative apex was excised, and octopine and Suc were extracted as described above. The quantity of ${ }^{14} \mathrm{C}$ extracted was determined by liquid scintillation counting. Based on the efficiency of ${ }^{14} \mathrm{C}$ transport, and the possibility that uptake and transport of octopine may be similar, octopine assays were set up with sufficient extract to provide final concentrations as high as $50 \mathrm{~nm}$, which is enough to saturate the assay and exceed the detection limit 50-fold.

\section{Sugar Analysis}

Sugar analysis was essentially as described (Turgeon and Gowan, 1992; Turgeon et al., 1993). In brief, plant tissue was excised, ground in liquid nitrogen, and extracted at $50^{\circ} \mathrm{C}$ in three changes of methanol:chloroform: water $(12: 5: 3[\mathrm{v} / \mathrm{v}])$, using 4 volumes per unit fresh weight for each extraction. Water was added to the extract (three parts water per five parts extract) to separate aqueous and organic phases, and after centrifugation, the aqueous phase was brought to dryness in a HetoVac (Jouan Nordic, Allerød, Denmark) centrifugal concentrator. Extracts were resuspended in water $(0.5$ $\mathrm{mL} g$ fresh weight ${ }^{-1}$ leaf tissue), and the neutral fraction was eluted from a column composed of, from bottom to top, AG1-X4 anion-exchange resin (Bio-Rad, Hercules, CA; hydrogen form converted to carbonate form; 1-mL bed volume per gram fresh weight tissue), polyvinylpolypyrolidone (0.5-mL bed volume per gram fresh weight tissue), and AG50W-X4 cation-exchange resin (Bio-Rad; hydrogen form; 1-mL bed volume per gram fresh weight tissue). The column was washed with three volumes of water, and the elutant concentrated to $0.5 \mathrm{~mL} \mathrm{~g}^{-1}$ of starting tissue. Sugars were resolved on Silica Gel GHL TLC plates (Analtech, Newark, DE) as described by Turgeon and Gowan (1992).

For labeling experiments, leaf tissue was photosynthetically labeled with ${ }^{14} \mathrm{C}$ essentially as described (Turgeon et al., 1993). In brief, plants were illuminated with approximately $1,000 \mu \mathrm{mol}$ photons $\mathrm{m}^{-2} \mathrm{~s}^{-1}$ from a $1,000-\mathrm{W}$ metal halide lamp. Leaves to be labeled were hermetically sealed in a plastic chamber and $20 \mu \mathrm{Ci}$ of ${ }^{14} \mathrm{CO}_{2}$ was delivered by mixing $20 \mu \mathrm{L}$ of $\mathrm{Na}_{2}{ }^{14} \mathrm{CO}_{3}$ (ICN, Costa Mesa, CA; $1 \mathrm{mCi} \mathrm{mL}^{-1} ; 56 \mathrm{mCi} \mathrm{mmol}$ ) with $50 \mu \mathrm{L}$ of $80 \%(\mathrm{v} / \mathrm{v})$ lactic acid in a syringe barrel open to the chamber. After $15 \mathrm{~min}$, the chamber was removed and photosynthesis continued for another $45 \mathrm{~min}$ in room atmosphere. Labeled sugars exported from C. blumei and tobacco leaves were detected from the petiole or stem-proximal section of the midrib, respectively. To prevent direct labeling, these tissues were excluded from the labeling chamber and enclosed in aluminum foil to prevent photosynthesis. For tobacco leaves, this was facilitated by removing the lamina along $4 \mathrm{~cm}$ of midrib adjacent to the stem. 
Quantitative measurements of sugar concentration and specific activity were as described (Bachmann and Keller, 1995; Haritatos et al., 1996). In brief, the carbohydrate masses were determined by HPLC with pulsed amperometric detection using cation-moderated partitioning chromatography (Benson BC-100 Ca-Carbohydrate column; $300 \times 7.8 \mathrm{~mm}$, fitted with a BC-100 guard column; Benson Polymeric, Reno, NV) with $50 \mathrm{mg} \mathrm{L}^{-1} \mathrm{Ca}$ EDTA as eluant (at $0.5 \mathrm{~mL} \mathrm{~min}^{-1}$ and $90^{\circ} \mathrm{C}$ ). For radio-HPLC, the chromatography system was linked to an on-line radio detector (Radiomatic 500TR Series flow scintillation analyzer, Packard, Zurich). Post-column addition of liquid scintillation cocktail (Flo-Scint A, Packard) occurred at a rate of 1.5 $\mathrm{mL} \min ^{-1}$.

\section{Distribution of Materials}

Upon request, all novel materials described in this publication will be made available in a timely manner for noncommercial research purposes, subject to the requisite permission from any third party owners of all or parts of the material. Obtaining any permissions will be the responsibility of the requestor.

\section{ACKNOWLEDGMENTS}

We thank Roisin C. McGarry and Edwin J. Reidel for reviewing the manuscript, Stephen C. Winans for suggesting that A. tumefaciens strain KYC16 could be used in a quantitative bioassay for octopine, and Randy $\mathrm{O}$. Wayne for use of equipment.

Received July 30, 2002; returned for revision September 25, 2002; accepted November 24, 2002.

\section{LITERATURE CITED}

Ausubel F, Brent R, Kingston RE, Moore DD, Seidmam JG, Smith JA, eds (1995) Short Protocols in Molecular Biology, Ed 3. John Wiley and Sons, Inc., New York

Bachmann M, Keller F (1995) Metabolism of the raffinose family oligosaccharides in leaves of Ajuga reptans L.: intercellular and intracellular compartmentation. Plant Physiol 109: 991-998

Becker D, Kemper E, Schell J, Masterson R (1992) New plant binary vectors with selectable markers located proximal to the left T-DNA border. Plant Mol Biol 20: 1195-1197

Beebe DU, Turgeon R (1992) Localization of galactinol, raffinose, and stachyose synthesis in Cucurbita pepo leaves. Planta 188: 354-361

Bennet AB, Damon S, Osteryoung K, Hewitt J (1986) Mechanisms of retrieval and metabolism following phloem unloading. In J Cronshaw, WJ Lucas, RT Giaquinta, eds, Phloem Transport. A.R. Liss, New York, pp 307-316

Burkle L, Hibberd JM, Quick WP, Kühn C, Hirner B, Frommer WB (1998) The $\mathrm{H}^{+}$-sucrose cotransporter NtSUT1 is essential for sugar export from tobacco leaves. Plant Physiol 118: 59-68

Cangelosi GA, Best EA, Martinetti G, Nester EW (1991) Genetic-analysis of Agrobacterium. Methods Enzymol 204: 384-397

Cho KY, Fuqua C, Martin BS, Winans SC (1996) Identification of Agrobacterium tumefaciens genes that direct the complete catabolism of octopine. J Bacteriol 178: 1872-1880

Dessaux Y, Petit A (1994) Opines as screenable markers for plant transformation. In SB Gelvin, RA Schilperoort, eds, Plant Molecular Biology Manual, Ed 2. Kluwer Academic, Norwell, MA, pp C3: 1-12

Fisher DB, Wu Y, Ku MSB (1992) Turnover of soluble proteins in the wheat sieve tube. Plant Physiol 100: 1433-1441

Fujimaki S, Fujiwara T, Hayashi H (2000) A new method for direct introduction of chemicals into a single sieve tube of intact rice plants. Plant Cell Physiol 41: 124-128

Geiger DR, Giaquinta RT, Sovonick SA, Fellows RJ (1973) Solute distribution in sugar beet leaves in relation to phloem loading and translocation. Plant Physiol 52: 585-589

Giaquinta RT (1983) Phloem loading of sucrose. Annu Rev Plant Physiol 34: 347-387

Grusak MA, Beebe DU, Turgeon R (1996) Phloem loading. In E Zamski, AA Schaffer, eds, Photoassimilate Distribution in Plants and Crops: SourceSink Relationships. Marcel Dekker, New York, pp 209-227
Haritatos E, Ayre BG, Turgeon R (2000) Identification of phloem involved in assimilate loading in leaves by the activity of the galactinol synthase promoter. Plant Physiol 123: 929-937

Haritatos E, Keller F, Turgeon R (1996) Raffinose oligosaccharide concentrations measured in individual cell and tissue types in Cucumis melo L. leaves: implications for phloem loading. Planta 198: 614-622

Holthaus U, Schmitz K (1991) Distribution and immunolocalization of stachyose synthase in Cucumis melo L. Planta 185: 479-486

Hsu FC, Kleier DA (1996) Phloem mobility of xenobiotics: VIII. A short review. J Exp Bot 47: 1265-1271

Husted S, Schjoerring JK (1995) Apoplastic pH and ammonium concentration in leaves of Brassica napus L. Plant Physiol 109: 1453-1460

Imlau A, Truernit E, Sauer N (1999) Cell-to-cell and long-distance trafficking of the green fluorescent protein in the phloem and symplastic unloading of the protein into sink tissues. Plant Cell 11: 309-322

Jefferson RA, Kavanagh TA, Bevan MW (1987) GUS fusions: $\beta$-glucuronidase as a sensitive and versatile gene fusion marker in higher plants. EMBO J 6: 3901-3907

Keller F, Pharr DM (1996) Metabolism of carbohydrates in sinks and sources: galactosyl-sucrose oligosaccharides. In E Zamski, AA Schaffer, eds, Photoassimilate Distribution in Plants and Crops: Source-Sink Relationships. Marcel Dekker, New York, pp 157-183

Kempers R, Prior DAM, van Bel AJE, Oparka KJ (1993) Plasmodesmata between sieve element and companion cell of extrafascicular stem phloem of Cucurbita maxima permit passage of $3 \mathrm{kDa}$ fluorescent probes. Plant J 4: 567-575

Kempers R, van Bel AJE (1997) Symplasmic connections between sieve element and companion cell in the stem phloem of Vicia faba L have a molecular exclusion limit of at least $10 \mathrm{kDa}$. Planta 201: 195-201

Kim M, Canio W, Kessler S, Sinha N (2001) Developmental changes due to long-distance movement of a homeobox fusion transcript in tomato. Science 293: 287-289

Knop C, Voitsekhovskaja O, Lohaus G (2001) Sucrose transporters in two members of the Scrophulariaceae with different types of transport sugar. Planta 213: 80-91

Kühn C, Franceschi VR, Schulz A, Lemoine R, Frommer WB (1997) Macromolecular trafficking indicated by localization and turnover of sucrose transporters in enucleate sieve elements. Science 275: 1298-1300

Loewus FA, Murthy PPN (2000) myo-Inositol metabolism in plants. Plant Sci 150: $1-19$

Lohaus G, Pennewiss K, Sattelmacher B, Hussmann M, Muehling KH (2001) Is the infiltration-centrifugation technique appropriate for the isolation of apoplastic fluid? A critical evaluation with different plant species. Physiol Plant 111: 457-465

Lohaus G, Winter H, Riens B, Heldt HW (1995) Further studies of the phloem loading process in leaves of barley and spinach: the comparison of metabolite concentrations in the apoplastic compartment with those in the cytosolic compartment and in the sieve tubes. Bot Acta 108: 270-275

Madore MA (1990) Carbohydrate metabolism in photosynthetic and nonphotosynthetic tissues of variegated leaves of Coleus blumei Benth. Plant Physiol 93: 617-622

Maille M (1983) A radiotechnique suitable for the detection of octopine synthesis in crown-gall tissues grown in vitro. Anal Biochem 134: 133-136

Marentes E, Grusak MA (1998) Mass determination of low-molecularweight proteins in phloem sap using matrix-assisted laser desorption/ ionization time-of-flight mass spectrometry. J Exp Bot 49: 903-911

Oparka KJ, Roberts AG, Boevink P, Santa Cruz S, Roberts L, Pradel KS, Imlau A, Kotlizky G, Sauer N, Epel B (1999) Simple, but not branched, plasmodesmata allow the nonspecific trafficking of proteins in developing tobacco leaves. Cell 97: 743-754

Oparka KJ, Turgeon R (1999) Sieve elements and companion cells: traffic control centers of the phloem. Plant Cell 11: 739-750

Thorpe MR, Minchin PEH (1996) Mechanism of long- and short-distance transport from sources to sinks. In E Zamski, AA Schaffer, eds, Photoassimilate Distribution in Plants and Crops: Source-Sink Relationships. Marcel Dekker, New York, pp 261-282

Turgeon R (1984) Efflux of sucrose from minor veins of tobacco leaves. Planta 161: 120-128

Turgeon R (1995) The selection of raffinose oligosaccharides as translocates in higher plants. In M Madore, WJ Lucas, eds, Carbon Partitioning and 
Ayre et al.

Source-Sink Interactions in Plants. American Society of Plant Physiologists, Rockville, MD, pp 195-203

Turgeon R (1996) Phloem loading and plasmodesmata. Trends Plant Sci 1: $418-423$

Turgeon R, Beebe DU, Gowan E (1993) The intermediary cell-minor-vein anatomy and raffinose oligosaccharide synthesis in the Scrophulariaceae. Planta 191: 446-456

Turgeon R, Gowan E (1990) Phloem loading in Coleus blumei in the absence of carrier-mediated uptake of export sugar from the apoplast. Plant Physiol 94: 1244-1249

Turgeon R, Gowan E (1992) Sugar synthesis and phloem loading in Coleus blumei leaves. Planta 187: 388-394
Turgeon R, Medville R, Nixon KC (2001) The evolution of minor vein phloem and phloem loading. Am J Bot 88: 1331-1339

van Bel AJE (1993) The transport phloem. Specifics of its functioning. Prog Bot 54: 134-150

van Bel AJE, Knoblauch M (2000) Sieve element and companion cell: the story of the comatose patient and the hyperactive nurse. Aust J Plant Physiol 27: 477-487

Xoconostle-Cazares B, Yu X, Ruiz-Medrano R, Wang HL, Monzer J, Yoo BC, McFarland KC, Franceschi VR, Lucas WJ (1999) Plant paralog to viral movement protein that potentiates transport of mRNA into the phloem. Science 283: 94-98 